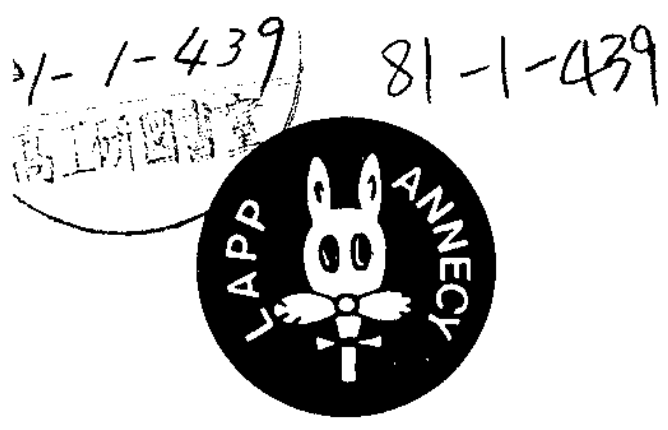

LAPP-TH-24

Ref.Th.2967-CERN

October 21,1980

\title{
A COSMOLOGICAL LOWER BOUND ON THE NEUTRON ELECTRIC DIPOLE MOMENT
}

\author{
John E11is, \\ CERN, Geneva, Switzerland \\ Mary K. Gaillard, \\ LAPP, Annecy-le-Vieux, France \\ D.V. Nanopoulos and Serge Rudaz, \\ CERN, Geneva, Switzerland
}

\section{A B S T R A C T}

We argue that in a wide class of grand unified theories diagrams similar to those generating baryon number in the early universe also contribute to renormalization of the CP-violating $\theta$ parameter of $\mathrm{QCD}$ and hence to the neutron electric dipole moment $d_{n}$. We then use the apparent baryon-to-photon ratio $\left(n_{B} / n_{\gamma}\right) \geqslant 1.3 \times 10^{-10}$ to deduce an order-of-magnitude lower bound on the neutron electric dipole moment: $\mathrm{d}_{\mathrm{n}} \geq 3 \times 10^{-28} \mathrm{e}-\mathrm{cm}$. Conversely the present experimental upper Iimit on $d_{n}$ implies $\left(n_{B} / n_{\gamma}\right) \leqslant 6 \times 10^{-7}$. We find as a corollary that there is not much scope for entropy generation after the creation of the baryon-antibaryon asymetry in the very early universe. 
After the experimental discovery that $C P$ is not conserved in the $\mathrm{K}^{0}-\overline{\mathrm{k}}^{\circ}$ system ${ }^{1)}$, many attempts were made to observe other CP-violating phenomena in elementary particle physics, such as an electric dipole moment of the neutron $d_{n}{ }^{2)}$. No other manifestations of $C P$ violation were found, and there has been a tendancy to consider CP nonconservation as a curiosity whose great physical significance was not obvious. However, it was realized ${ }^{3)}$ soon after its discovery in the $\mathrm{K}^{0}-\overrightarrow{\mathrm{k}}^{0}$ system that $\mathrm{CP}$ violation would be an essential ingredient in any attempt to generate a net cosmological baryon asymmetry starting from an initially symmetric universe. This realization ${ }^{3)}$ has recently been fleshed out by grand unified theories (GUTs) ${ }^{4}$ ), which seen to provide a realistic framework for calculating baryon number generation in the very early universe when the temperature was of order $10^{15} \mathrm{GeV}^{5}$ ). The natural question then arises whether there is any connection between the CP violation seen at the relatively low energies presently accessible with accelerators, and the high energy CP violation believed to be responsible for baryon generation. This question has so far been answered in the negative ${ }^{6}$, at least in so far as a direct connection between the baryon-to-entropy ratio ( $\left.\pi_{B} / a_{\gamma}\right)$ and the $\varepsilon$ parameter of the $\mathrm{x}^{\circ}-\overline{\mathrm{K}}^{\circ}$ system is concerned. Even in the most constrained minimal GUT the CP violating phase encountered in the $\mathbb{K}^{\circ}-\overline{\mathrm{K}}^{\circ}$ system is not the dominant one in the cosmological generation of baryon number ${ }^{7)}$, and it seems unlikely that a direct connection would be recovered in a non-minimal GUT. Could there be any connection between $\left(\pi_{B} / n_{\gamma}\right)$ and any other low-energy manifestation of CP-violation such as the neutron electric dipole moment?

In this paper we argue that in a wide class of GUTs the class of diagrams that generates $\left(n_{B} / n_{\gamma}\right)$ also contributes indirectly to the neutron electric dipole moment ${ }^{8)}$. Allowing for other possible contributions to $d_{n}$, and for the possibility that extra entropy may be generated during ${ }^{9}$ or after ${ }^{10}$ ) the epoch of baryon number generation, we obtain the order of magnitude inequality

$$
d_{n} \geq 2.5 \times 10^{-18}\left(n_{B} / n_{Y}\right) e-c m .
$$

Taking as an astrophysical 1 imit 11$)$ the present apparent value of $\left(n_{B} / n_{\gamma}\right) \geqslant$ $1.3 \times 10^{-1}$ a we deduce that the neutron electric dipole moment cannot be smaller than about $3 \times 10^{-28} \mathrm{e}-\mathrm{cm}$. Conversely, the present upper limit ${ }^{2)}$ on $\mathrm{d}_{\mathrm{n}}$ of $1.6 \times 10^{-24} \mathrm{e}-\mathrm{cm}$ implies an upper limit on the baryon-to-entropy ratio of order

$$
\left(n_{B} / n_{\gamma}\right) \leq 6 \times 10^{-7}
$$

which is consistent with our cosmological knowledge ${ }^{11}$ ). The bound (1) can be applied to the $\left(n_{B} / n_{\gamma}\right)$ generated primordially by GUTs, and if a lot of entropy 
has been generated during ${ }^{9)}$ or after ${ }^{10)}$ the generation of baryon number, then the inequality (1) becomes strict, and $d_{n}$ approaches the experimental upper limit of $1.6 \times 10^{-24} \mathrm{e}-\mathrm{cm}$. This upper limit on $d_{n}$ means that not more than a factor of $5 \times 10^{3}$ or so of entropy could have been generated during or after the cosmological generation of baryon number.

We start our derivation of these results by recalling certain elements in the estimation of the neutron electric dipole moment. This receives a contribution from conventional weak interaction perturbation theory ${ }^{12}$ ), which has been estimated to be of order

$$
\Delta d_{\mathrm{n}} \approx 10^{-30 \pm 1} \mathrm{e}-\mathrm{cm}
$$

in the minimal 6-quark Kobayashi-Maskawa ${ }^{13)}$ version of the Weinberg-Salam model with just one complex Higgs doublet. Another contribution ${ }^{14)}$ to $d_{n}$ could come from a non-zero value of the QCD vacuum parameter $\theta$ :

$$
\Delta \mathrm{d}_{\mathrm{n}} \approx 4 \times 10^{-16} \theta \mathrm{e}-\mathrm{cm}
$$

No one has a convincing estimate of $\theta$. It is a completely arbitrary parameter within the context of pure $Q C D$, subject to renormalization by the CP-violating non-strong interactions. We will argue ${ }^{8)}$ that GUT diagrams analogous to those generating $\left(n_{B} / n_{Y}\right)$ also contribute to the renormalization of $\theta$, and that they give an order-of-magnitude lower bound to the plausible value of $\theta$ to be inserted in equation (4).

Renormalization of the QCD $\theta$ vacuum parameter can arise from the necessity of redefining the quark mass matrix in each order of perturbation theory in the non-strong interactions ${ }^{15)}$. It has been argued ${ }^{16)}$ that $\theta$ renomalization can also arise from the direct generation of a counter-term $\approx F_{\mu \nu} \tilde{F}^{\text {jV }}$ when doing non-strong perturbation theory in the presence of strong non-perturbative effects. Any such contribution would have a diagrammatic structure similar to the quark mass matrix renormalization we consider here. We write ${ }^{17)}$ the bare inverse quark propagator as

$$
S_{0}^{-1}=p-M
$$

and denote by $\Sigma(p)$ the sum of irreducible weak/electromagnetic contributions to the quark propagator. It has the Dirac decomposition

$$
\Sigma(p)=A p L+B p R+M C L+D M R: A=A^{+}, B=B^{+}, C=D^{+}
$$


The renormalized mass matrix $M^{\text {Ien }}$ is the coefficient of $I$ in a Dirac decomposition of the full inverse quark propagator $p-M-\Sigma(p)$ after it has been renormalized. We then have

$$
\begin{aligned}
\delta \theta & =\operatorname{Arg} \operatorname{det} M^{\text {Ien }} \\
& =\operatorname{Im} \operatorname{Tr} C+O\left(C^{2}\right)
\end{aligned}
$$

If we introduce a running quark mass $\mathrm{M}^{\text {ren }}(\mu)$ through the renormalization prescription

$$
\left[S^{\operatorname{ren}}(p)\right]_{p^{2}=-\mu^{2}}^{-1}=p-M^{\operatorname{ren}}(\mu)
$$

then the $\theta$ parameter also becomes ${ }^{17)}$ a function of $\mu$ with

$$
\mu \frac{\partial \theta}{\partial \mu}=\beta_{\theta}=\mu \frac{\partial}{\partial \mu}\left(\arg \operatorname{det} M^{\text {Ien }}\right) \approx \mu \frac{\partial}{\partial \mu} \operatorname{In} \operatorname{Ir} C+o\left(C^{2}\right)
$$

This formulation can easily be adapted to accomodate the incorporation of QCD into a GUT. The renormalization prescription ( 9 ) is extended to all fermions, quarks and leptons, and the $\theta$ parameter of the unbroken Grand Unification group will be renormalized as in $(10)$ when $\mu \gg m_{x}$. When $\mu \lesssim m_{x}$ the different unbroken non-Abelian subgroups will have their $\theta$ parameters renormalized differently, since they arise from redefining different submatrices of the full fermion mass matrix $\mathrm{M}^{\mathrm{ren}}$. The QCD $\theta$ parameter appearing in calculations of the neutron electric dipole moment is presumably the renormalized value at some typical hadronic momentum scale of order $1 \mathrm{GeV}$. It can be written in the form

$$
\theta_{\mathrm{QCD}}(1 \mathrm{GeV}) \simeq \delta \theta_{\mathrm{KM}}+\delta \theta_{\mathrm{GUT}}+\theta_{\mathrm{TOE}}
$$

where $\delta \theta_{K M}$ denotes the renormalization of $\theta$ at scales $\mu \ll m_{x}$ due to the weak interactions alone, $\delta \theta_{\mathrm{GUT}}$ denotes the renormalization of $\theta$ at scales

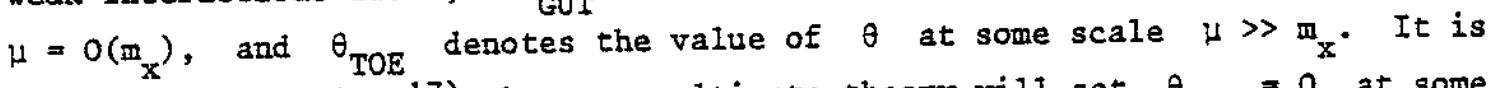
attractive tc believe 17 ) that some ultimate theory will set $\theta_{\text {TOE }}=0$ at some "relaxation" scale $>m_{x}$, perhaps at the Planck mass.

The general structure of the leading contributions to $\theta$ renormalization is shown in fig. la. It takes the form of a one particle irreducible left-right transition matrix $F_{1}$, which should be premultiplied by $\mathrm{M}^{-1}$ to obtain the matrix $C$ defined in equation (8);

$$
C=M^{-1} F_{1}
$$


Since the fermion mass matrix $M$ is generated by a single Higgs field ${ }^{18)}$, we know that

$$
\mathrm{M}=\mathrm{H}_{\mathrm{m}} \mathrm{v}
$$

where $H_{m}$ is the lowest order coupling matrix of the Higgs m which generates fermion masses, and $v$ is its vacuum expectation value. The matrix $H_{m}$ must be non-singular and hence have an inverse $\mathrm{H}_{\mathrm{m}}^{-1}$, because all the quark masses are non-zero. Combining equations (12) and (13) we get

$$
C=\frac{1}{v} \mathrm{E}_{\mathrm{m}}^{-1} F_{1}
$$

But we also know that the left-right transition matrix $F_{1}$ is related to a contribution $\mathrm{H}_{1}$ to the full m-Higgs coupling matrix

$$
\mathrm{F}_{3}=\mathrm{H}_{2} \mathrm{v}
$$

Combining equations (14) and (15) we get

$$
\mathrm{C}=\mathrm{H}_{\mathrm{m}}^{-1} \mathrm{H}_{1}
$$

and hence

$$
\delta \theta=\operatorname{Im} \operatorname{Tr} C+\ldots \approx \operatorname{Im} \operatorname{Tr}\left(H_{m}^{-1} H_{1}\right) .
$$

It is known ${ }^{17)}$ that $\delta \theta_{\mathrm{KM}}=O\left(10^{-16}\right)$ is rather sma11. We do not know what the original $\theta_{\text {TOE }}$ is, or why, but it seems reasonable to suppose that in order of magnitude

$$
\theta_{\mathrm{QCD}}(1 \mathrm{GeV}) \geq \delta \theta_{\mathrm{GUT}}
$$

We will now argue ${ }^{8)}$ that in a large class of GUTs the dominant contributions to baryon number generation have a diagramatic structure related to that of $A_{3}$ in fig. 1b.

Most GUTs ${ }^{5)}$ envisage that baryon number is generated by $\mathrm{C}$ - and CP-violating asymetries in the decays of particles with masses $O\left(\mathrm{~m}_{X}\right)$, which result in a net baryon asymetry $\Delta B$ per heavy particle decay. In the absence of any unexpected suppression factor or extra entropy generation, these would give

$$
\frac{\mathfrak{n}_{B}}{\mathfrak{n}_{\gamma}} \approx\left(10^{-1} \text { to } 10^{-2}\right) \Delta B
$$

The heavy particles could either be vector bosons, Higgs bosons or fermions. It has been pointed out that vector bosons are unlikely to make as large a contribution to $\left(n_{B} / n_{\gamma}\right)$ as Higgs bosons, because CP-violating asymetries generally occur 
in higher order in gauge boson decays ${ }^{7)}$. Also it is more difficult to wipe out or dilute via $2 \leftrightarrow 2$ interactions any CP-violating asymetries from Higgs decays in models with reducible multiplets of fermions ${ }^{19}$ ), because the Higgs-fermion couplings are generally smaller than the gauge coupling. There is no good reason why heavy fermion decays should not make an important contribution to $\left(n_{B} / n_{\gamma}\right)$, but we will follow the bulk of the literature in disregarding them ${ }^{20}$ ). If our forthcoming bound on $d_{n}$ in terms of $n_{B} / n_{\gamma}$ is violated, this would be one of the first assumptions to be questioned: perhaps $d_{n}$ will give us indirect evidence for the existence of heavy fermions with masses $O\left(m_{x}\right)$ ?

Let us call $d$ one of the Higgs fields making a substantial contribution to the baryon number through its decays. The generic structure of diagrams $I_{1}$ contributing to CP-violating and baryon number generating asymetries in d Higgs boson decays is shown in fig. 2a. It gives an order of magnitude:

$$
\Delta B \approx \frac{\operatorname{Im} \operatorname{Tr}\left(I_{3}\right)}{\operatorname{Tr}\left(H_{d} H_{d}^{+}\right)}=\frac{\operatorname{Im} \operatorname{Tr}\left(a_{d} b_{d}^{\dagger}\right)}{\operatorname{Tr}\left(H_{d} H_{d}^{\dagger}\right)}
$$

where $\mathrm{H}_{\mathrm{d}}$ is the lowest order coupling matrix of the $d$ Higgs. The $d$ Higgs and the $m$ Higgs are not necessarily in the same irreducible multiplet of the GUT. For convenience, let us work in a "decay basis" for the Higgs fields in which the d Higgs is a pure state, along with its partners in an irreducible GUT multiplet, though these are not in general wass eigenstates. We will always be doing calculations in a mass or momentum scale close to the grand unification scale, so that the Higgs and other couplings are always GUT invariant in lowest order. The $m$ Higgs is a linear superposition $U_{j m}$ of Higgs fields $j$ which will in general include the partners of the $d$ Higgs in an irreducible GUT multiplet. Therefore in the decay basis introduced earlier, the coupling matrix for the in Higgs can be written as

$$
\mathrm{H}_{\mathrm{m}}=\sum_{j} \mathrm{H}_{\mathrm{j}} \mathrm{U}_{\mathrm{jm}}: \mathrm{U}_{\mathrm{dm}} \neq 0 \text { in general. }
$$

We are now in a position to rewrite the imaginary part of the trace in equation (20) in such a way that an analogous trace manifestly contributes to the renormalization of $\theta$. We have

$$
\text { In } \operatorname{Tr}\left(a_{d} b_{d}^{\dagger}\right)=\operatorname{Im} \operatorname{Ir}\left(H_{m}^{-1} H_{m} a_{d} b_{d}^{\dagger}\right)
$$

and in general there is a diagram contributing to the mass Higgs coupling matrix $\mathrm{H}_{1}$ which has the topological structure indicated in fig. $2 \mathrm{~b}$, and has a similar trace to that in equation (22): 
$\operatorname{Im} \operatorname{Tr}(f i g .2 b)=I m \operatorname{Tr}\left(H_{m}^{-1} H_{m} a_{d} b_{d}^{\dagger}\right) \times\left|U_{d m}\right|^{2}$

This is clearly a contribution to $\operatorname{Im} \operatorname{Tr}\left(\mathrm{H}_{\mathrm{m}}^{-1} \mathrm{H}_{1}\right)$, and in general we have no reason to expect that $\left|\mathrm{U}_{\mathrm{dm}}\right|^{2}<<1$. Therefore we have in order of magnitude (barring bizarre cancellations, but we never promised you a rose garden):

$$
\begin{aligned}
\delta \theta_{\text {GUT }} & \approx \operatorname{Im} \operatorname{Tr}\left(B_{\mathrm{m}}^{-1} \mathrm{H}_{\mathrm{l}}\right) \geq \operatorname{Im} \operatorname{Tr}\left(\mathrm{H}_{\mathrm{m}}^{-1} \mathrm{H}_{\mathrm{m}} \mathrm{a}_{\mathrm{d}} \mathrm{b}_{\mathrm{d}}^{\dagger}\right)\left|\mathrm{J}_{\mathrm{dm}}\right|^{2} \\
& \approx \operatorname{Im} \operatorname{Tr}\left(\mathrm{a}_{\mathrm{d}} \mathrm{b}_{\mathrm{d}}^{\dagger}\right) \approx \operatorname{Tr}\left(\mathrm{H}_{\mathrm{d}} \mathrm{B}_{\mathrm{d}}^{+}\right) \Delta \mathrm{B} \geq 10 \operatorname{Tr}\left(\mathrm{H}_{\mathrm{d}} \mathrm{B}_{\mathrm{d}}^{\dagger}\right)\left(\frac{\mathrm{n}_{\mathrm{B}}}{\mathrm{R}_{\gamma}}\right) .
\end{aligned}
$$

If we guess as an order of magnitude that

$$
\operatorname{Tr}\left(\mathrm{H}_{\mathrm{d}} \mathrm{H}_{\mathrm{d}}^{+}\right) \approx \sqrt{2} \mathrm{G}_{\mathrm{F}} \mathrm{m}_{t}^{2} \geq 6 \times 10^{-4}
$$

by taking $m_{t} \geq 18 \mathrm{GeV}$ at present energies and rescaling to an effective $m_{t} \geq 6 \mathrm{GeV}$ at grand unification, then we finally obtain from equations (18) and (25) that

$$
\theta_{\mathrm{QCD}}(1 \mathrm{GeV}) \geq 6 \times 10^{-3}\left(\frac{\mathrm{n}_{\mathrm{B}}}{\mathrm{n}_{Y}}\right) .
$$

When combined with equation (4) this gives us the advertized result of equation (1). One would be tempted to increase this lower bound if the lower limit on the mass of the top quark were significantly improved, though it should be borne in mind that equation $(26)$ is only approximate, since $m_{t}$ is only related directly to the II Higgs couplings and not directly to the d Higgs couplings appearing in equations (25) and (26). A plausible guess is that $\operatorname{Tr}\left(\mathbb{H}_{\mathrm{d}} \mathrm{E}_{\mathrm{d}}^{+}\right) / 4 \pi \leq 0\left(\alpha_{G U T}\right)$ $=0(1 / 42)$.

Before discussing the phenomenology of this result, let us first illustrate our line of argument by two specific examples. Our first unrealistic example ${ }^{7)}$ is the minimal SU(5) model with a single $\underline{5}$ of Higgs fields, in which the d and $m$ Higgses are in an irreducible multiplet. The minimal trace contributing to baryon generation is eighth order in the Higgs couplings, as illustrated in fig. 3a. A trace of similar form also contributes ${ }^{17)}$ to $\delta \theta$, as illustrated in fig. 3b. This is an example of the crucial inequality (25), which is in fact not saturated in minimal $\mathrm{SU}(5)$. Fig. $3 \mathrm{~b}$ is not the lowest order contribution to $\theta$ renormalization, as there are contributions to $\delta \theta_{\mathrm{KM}}$ in this model which are of order (mixing angles) $\times \alpha^{2} \alpha_{Q C D}^{2}\left(m_{q} / m_{w}\right)^{4}$ and hence much larger than the (mixing angles $) \times \alpha^{4}\left(\mathrm{~m}_{\mathrm{q}} / \mathrm{m}_{w}\right)^{8}$ which we get from fig. $3 \mathrm{~b}$. Hence minimal $\mathrm{SU}(5)$ respects the inequality ( 1 ), though it is unrealistic in that it predicts a value for $\left(n_{B} / n_{\gamma}\right)$ which is several orders of magnitude too smal1 ${ }^{8}$. 
A second, possibly realistic, model is one with two $\underline{5}^{\prime}$ s of scalar fields ${ }^{21}$ ). The $m$ Higgs is in an irreducible representation with some linear combination of the d Higgs and another $S U(3)_{c}$ triplet of scalars orthogonal to it. The fourth order diagram of fig. 4 a contributes to baryon number generation via the decay $\overrightarrow{\mathrm{H}} \rightarrow 10+10$. (The diagram with all arrows reversed and cut on the right hand corner gives an additional contribution via the decay $\overline{\mathrm{H}} \rightarrow 5+\overline{10}$. Although the relevant traces of coupling matrices for these two contributions are complex conjugates of one another, they do not cancel because they have different net

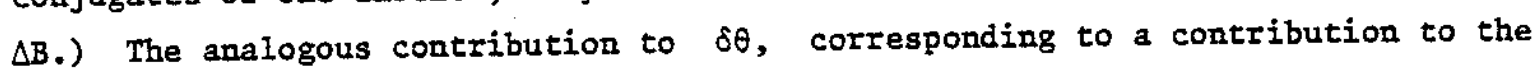
mass matrix of charge $-1 / 3$ quarks, is shown in fig. $4 \mathrm{~b}$. There are of course many other fourth order contributions to $\delta \theta$ in this model. The plethora of parameters arising from the arbitrary Yukawa couplings of the second scalar multiplet, orthogonal to the one containing the $m$ Higgs, hinders a quantitative estimate, but we have checked explicitly that there is no trivial cancellation of fig. 3b. Specifically, there is a contribution to the charge $-1 / 3$ mass matrix which has as coupling trace the complex conjugate of fig. $3 b$, but with a different loop structure and therefore a different coefficient a priori, and the contribution to the charge $2 / 3$ mass matrix with the same loop structure as fig. $3 b$ also has the same phase.

Let us now discuss the phenomenological implications of the inequality (1). It has been inferred ${ }^{(1)}$ indifectly from observations of X-ray emission from galactic dusters that

$$
\left(n_{B} / n_{Y}\right) \geq 1.3 \times 10^{-10}
$$

When inserted into equation (1) this yields

$$
\mathrm{d}_{\mathrm{n}} \geq 3 \times 10^{-28} \mathrm{e}-\mathrm{cm}
$$

which is considerably below the present ${ }^{2)}$ experimental limit of $1.6 \times 10^{-24} \mathrm{e}-\mathrm{cm}$, and beyond the likely sensitivity of forthcoming experiments. However, it is considerably larger than the $10^{-30} \mathrm{e}-\mathrm{cm}$ expected from the six-quark KobayashiMaskawa model alone ${ }^{12)}$. If a neutron electric dipole moment is detected at a level considerably higher than (3), this does not necessarily mean that the $K-M$ weak interaction model ${ }^{13}$ ) is wrong, but may only indicate that there is an additional high-energy source of CP-violation. In contrast to the $\mathrm{K}_{1}-\mathrm{K}_{2}$ system, the $d_{n}$ is unique in being sensitive to this high energy CP-violation, which may well be isomorphic with that responsible for baryon number generation.

It is worth noting that one way in which the inequality could be obeyed strictly, rather than barely saturated, would be if a sizeable amount of entropy 
has been generated during ${ }^{9}$ or after ${ }^{10}$ ) the generation of a baryon asymmetry. As discussed quantitatively in ref. 9, this can happen if the masses of the superheavy decaying particles are too small, or their coupling constants too large, in which case $2 \leftrightarrow 2$ interactions can wash out part of the baryon asymmetry. Another possibility raised recentiy ${ }^{10}$ ) is that a large amount of entropy (a factor $M$ which could be of order $10^{5}$ or $10^{6}$ if symetry breaking is due to radiative corrections ${ }^{22}$ ) may be generated during the Weinberg-Salam phase transition because of a period of exponential expansion during an epoch of supercooling. In this case we have

$$
1.6 \times 10^{-24} \mathrm{e}-\mathrm{cm} \geq d_{n} \geq M \times 3 \times 10^{-28} \mathrm{e}-\mathrm{cm}
$$

and we would infer that $M \leq 5 \times 10^{3}$. This bound may already cause problems for the scenario of Weinberg-Salam symetry breaking through radiative corrections ${ }^{22}$ ). These problems would become acute if the present bound on the neutron electric dipole moment is improved significantly without it being found. Furthermore, it would be tempting to increase the lower bound on the right-hand-side of equation (30) if the present lower 1imit of $18 \mathrm{GeV}$ on the mass of the heaviest quark is increased. Equation (30) could even be used to give an order of magnitude upper bound on the mass of the heaviest quark. At present this bound is of order $1 \mathrm{TeV}$, but it could be significantly improved by a more stringent upper limit on $d_{n}$, or if one became sure that $M>1$.

Although the cosmological lower bound (1) on the neutron electric dipole moment is not on a watertight theoretical basis, we feel that it is sufficiently welifounded to be taken seriously as a phenomenological constraint. This constraint is already non-trivial, and will become more significant as our knowledge of $d_{n}$ ' quark masses and $\left(\mathfrak{n}_{B} / n_{\gamma}\right)$ improves.

ACKNOWLEDGEMENTS

We thank G. Cocconi for his interest and encouragement. 


\section{REFERENCES}

1) J.H. Christensen, J.W. Cronin, V.I. Fitch and R. Turlay, Phys. Rev. Letters 13 (1964) 138.

2) I.S. Altarev et al., Pis'ma Zh. Eksp. Teor. Fiz. 29 (1979) 794 ;

W.B. Dress, P.D. Miller, J.M. Pendlebury, P. Perrin and N.F. Ramsey, Phys. Rev. D15 (1977) 9; see also, N.F. Ramsey, Phys. Reports 43 (1978) 409.

3) A.D. Sakharov, Pis'ma Zh. Eksp. Teor. Fiz. 5 (1967) 32.

4) J.C. Pati and A. Salam, Phys. Rev. Letters 31 (1973) 661 and Phys. Rev. D8 (1973) 1240;

E. Georgi and S.L. Glashow, Phys. Rev. Letters 32 (1974) 438.

5) M. Yoshimura, Phys. Rev. Letters 41 (1978) 281, 42 (1979) 746(E);

A. Yu Ignatiev, N.V. Krasnikov, V.A. Kuzmin and A.N. Tavkhelidze, Phys. Letters 76B (1978) 436 ;

S. Dimopoulos and L. Susskind, Phys. Rev. DI8 (1978) 4500;

D. Toussaint, S.B. Treiman, F. Wilczek and A. Zee, Phys. Rev. D19 (1979) 1036;

J. ElIis, M.K. Gaillard and D.V. Nanopoulos, Phys. Letters $80 B$ (1979) 360 , 82B (1979) $464(E)$;

S. Weinberg, Phys. Rev. Letters 42 (1979) 850;

A.D. Sakharov, Zh. Eksp. Teor. Fiz. 76 (1979) 1172;

S. Dimopoulos and L. Susskind, Phys. Letters 81B (1979) 416;

M. Yoshimura, Phys. Letters 88B (1979) 294;

D.V. Nanopoulos and S. Weinberg, Phys. Rev. D20 (1979) 2484;

S. Barr, G. Segrè and A. Weldon, Phys. Rev. D20 (1979) 2494.

6) J. E1lis, M.K. Gaillard and D.V. Nanopoulos, CERN-LAPP preprint, Ref.Th.2858CERN and LAPP-TH-19, in "Unification of the Fundamental Particle Interactions", Proc. of the Europhysics Study Conference, Erice (Plenum Press, 1980) P. 461 .

7) J. Ellis, M.K. Gaillard and D.V. Nanopoulos, Ref. 5;

see also J. Ellis, M.R. Gaillard and D.V. Nanopoulos, Phys. Letters 88B (1979) 320 .

8) This connection is implicit in the comparison of J. Ellis, M.K. Gaillard and D.V. Nanopoulos, Ref. 5, with section 5 of J. Ellis and M.K. Gaillard, Nuci. Phys. B150 (1979) 141, and explicit in M.R. Gaillard, contribution to the XXth International Conference on High Energy Physics, Madison, July 1980 (to appear in the Proceedings; report Fermilab-Conf-80/65-THY).

9) E.W. Kolb and S. Wolfram, Phys. Letters $91 \mathrm{~B}$ (1980) 217 and Nucl. Phys. 8172 (1980) 224;

J.N. Fry, K.A. Olive and M.S. Turner, Enrico Fermi Institute preprint 80-07 $(1980)$.

10) E. Witten, Harvard University preprint HUTP-80/AO40 (1980);

A.z. Guth and E.J.Weinberg, Phys. Rev. Letters 45 (1980) 1131 ;

M. Sher, U.C. Santa Cruz preprint UCSC $80 / 132$ (1980).

11) D.N. Schramm and G. Steigman, Bartol Research Foundation preprint BA-80-19, submitted to Ap. J.

12) J. Ellis, M.K. Gaillard and D.V. Nanopoulos, Nucl. Phys. B109 (1976) 213;

E.P. Shabalin, Yad. Fiz. 28 (1978) 151;

B.F. Morel, Nucl. Phys. B157 (1979) 23;

J.Ellis and M.K. Gaillard, Ref. 8;

D.V. Nanopoulos, A. Yildiz and P.H. Cox, Phys. Letters 87B (1979) 53 and Ann. Phys. (N.Y.) 127 (1980) 1 . 
13) M. Kobayashi and K. Maskawa, Prog. Theor. Phys. 49 (1973) 652.

14) V. Baluni, Phys. Rev. D19 (1979) 2227;

R.J. Crewther, P, di Vecchia, G. Veneziano and E. Witten, Phys. Letters 88B (1979) 123, 91B (1980) $487(E)$.

15) S. Weinberg, Phys. Rev. Letters 40 (1978) 223.

16) F. Wilczek, Phys. Rev. Letters 40 (1978) 279;

C.G. Han, Phys. Rev. D20 (1979) 996;

H. Georgi, T.N. Tomaras and A. Pais, Harvard University preprint HUTP-80/A046 (1980).

M. Claudson, Harvard University preprint HUTP-80/A061 (1980).

17) J. Ellis and M.K. Gaillard, Ref. 8.

18) H. Georgi and D.V. Nanopoulos, Phys. Letters 82B (1979) 95.

19) S.B. Treiman and F. Wilczek, Phys. Letters 95B (1980) 222.

20) See however R. Barbieri, A. Masiero and D.V. Nanopoulos, CERN preprint TH-2966 (1980) and references therein.

21) A. Yu Ignatiev, V.A. Kuzmin and M.E. Shsposhnikov, Pis'ma Zh. Eksp. Theor. Fiz. 30 (1979) 726 ;

A. Yildiz and P.H. Cox, Phys. Rev. D21 (1980) 906;

see also D.V. Nanopoulos and S. Weinberg, Ref. 5.

22) S. Coleman and E.J. Weinberg, Phys. Rev. D7 (1973) 1888;

E. Gildener and S. Weinberg, Phys. Rev. D13 (1976) 3333;

S. Weinberg, Phys. Lettexs 82B (1979) 387;

J. Ellis, M.K. Gaillard, D.V. Nanopoulos and C.T. Sachrajda, Phys. Letters 83B (1979) 339. 
FIGURE CAPTIONS

Fig. 1 (a) The one-particle irreducible left-right transition matrix $F_{1}$, which is related to (b) the Higgs-fermion-fermion coupling matrix $H_{1}$.

Fig. 2 (b) The generic structure of interference diagrams $I_{I}$ in Higgs decay which may generate a net baryon asymmetry, and (b) a diagram of analogous structure which contributes to $\delta \theta_{\mathrm{GUT}}$ and is expected to be of comparable order of magnitude. The dotted lines are cuts indicating sums (traces) over initial and final state particles.

Fig. 3 (a) A typical $8^{\text {th }}$ order contribution to $I_{1}$ in minimal $S U(5)$, and (b) a contribution to $\delta \theta_{\text {GUT }}$ which has the same algebraic structure of a product of Higgs-fermion-fermion vertices. The straight lines $\longrightarrow$ are fermions in a 10 of $s U(5)$ and the zigzag lines niw are fermions in a. $\underline{5}$ of $\mathrm{sU}(5)$.

Fig. 4 (a) A fourth order contribution to $I_{1}$ in an $S U(5)$ model with two scalar 5-plets, and (b) a contribution to $\delta \theta_{\text {GUT }}$ with the same algebraic structure. Notation is as in Fig. 3 except that $-\rightarrow \cdot-$ denotes a Higgs which is distinct from the decaying d Higgs. 


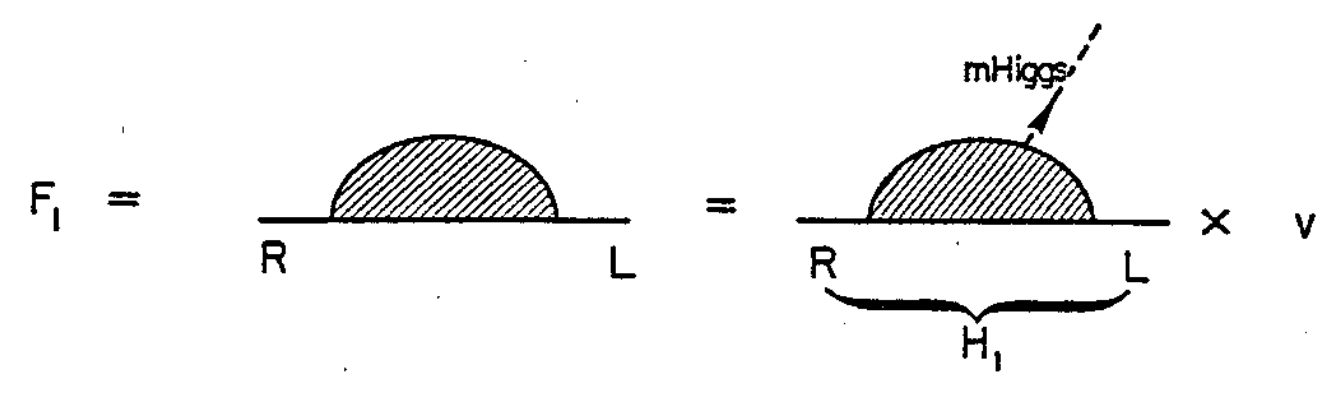

(a)

(b)

Fig. 1

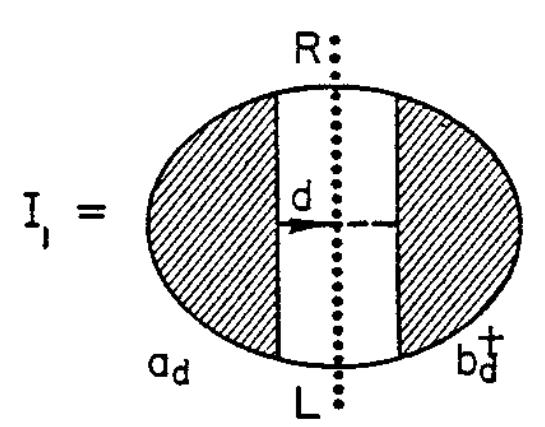

(a)

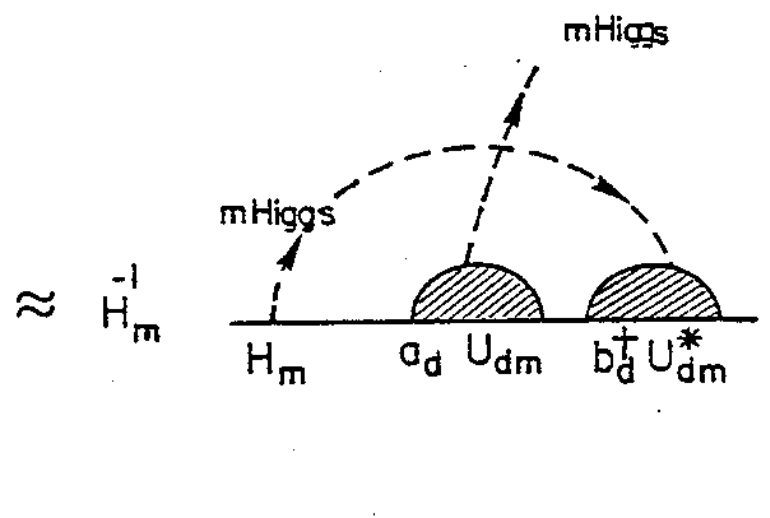

(b)

Fig. 2 


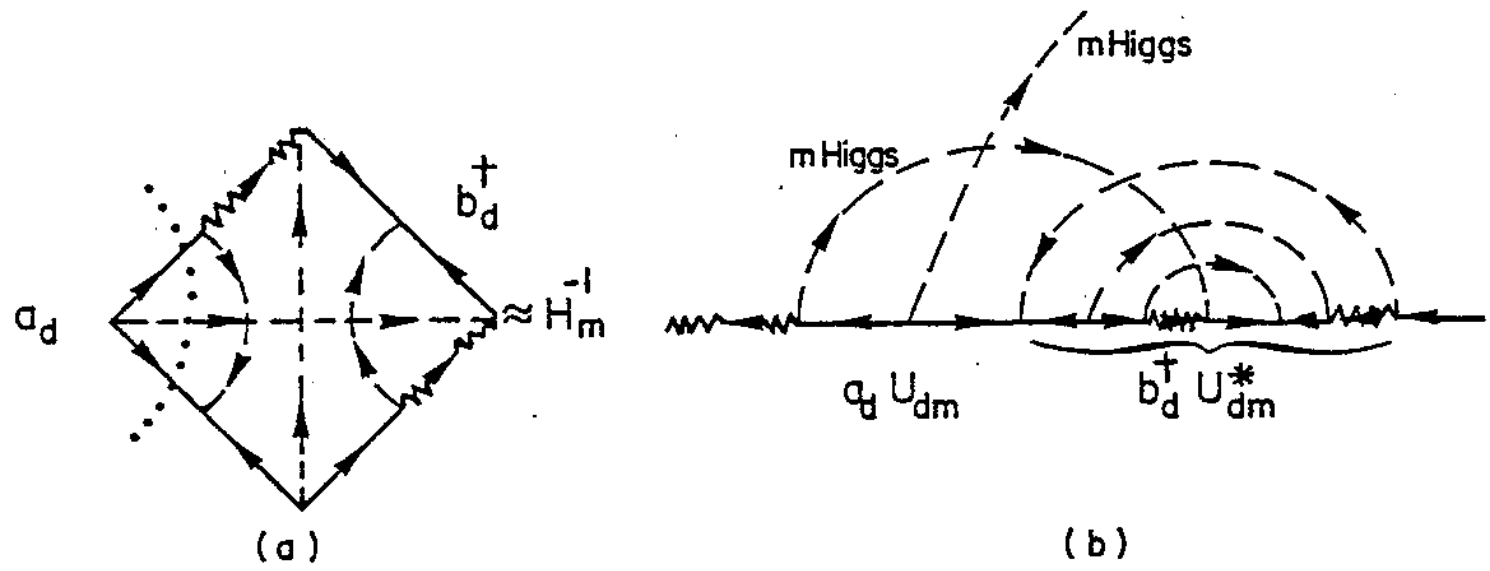

Fig. 3

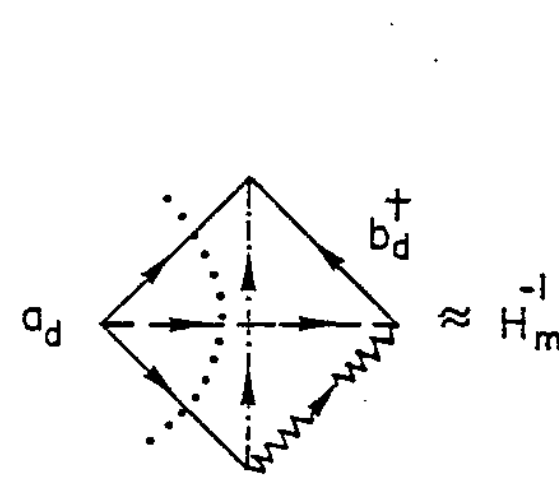

(a)

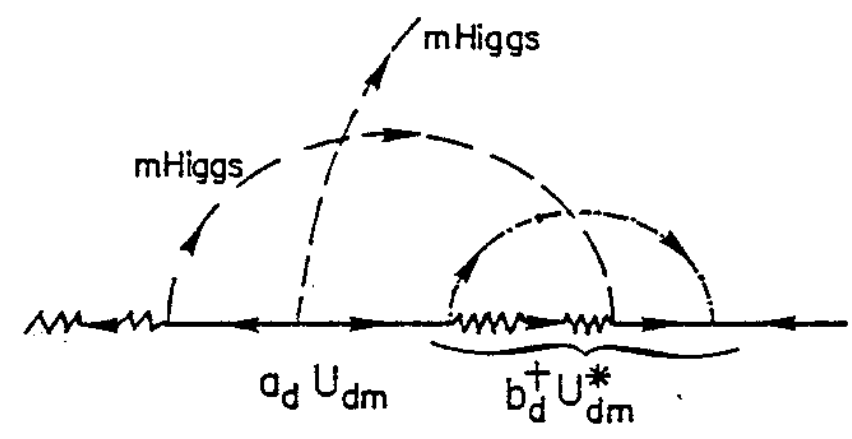

(b)

Fig. 4 